\title{
ESTABLECIMIENTO DE UN CEPARIO DE Helicobacter pylori, AISLADO DE BIOPSIAS GÁSTRICAS, ÚLCERA PÉPTICA, CÁNCER GÁSTRICO Y PERSONAS ASINTOMÁTICAS
}

\section{ESTABLISHMENT OF A STRAIN COLLECTION OF Helicobacter pylori ISOLATED FROM GASTRIC BIOPSY, PEPTIC ULCER, GASTRIC CANCER AND ASYMPTOMATIC PEOPLE}

\author{
Sebastián Rojas Lara $\bullet$ Julián Pineda $\bullet$ Diana Amarillo $\bullet$ Carlos Barragán ${ }^{1}$ Martín Bayona Rojas $\bullet$

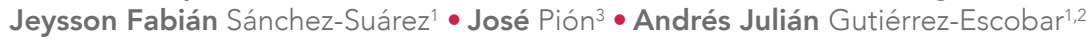

\section{RESUMEN}

Helicobacter pylori es un microorganismo que se estima infecta entre el 50 y $70 \%$ de la población general en países en vía de desarrollo y su infección se asocia a factores biopsicosociales. Actualmente se conoce la relación patogénica de la bacteria con gastritis, ulcera péptica e incluso cáncer gástrico y respecto a esta última patología, la OMS en 1994 la declaró como agente carcinogénico tipo I. En Colombia existen pocas colecciones caracterizadas microbiológica y clínicamente de éste microorganismo. El presente trabajo determinó las condiciones microbiológicas para el establecimiento de un cepario de $\mathrm{H}$. pylori. Se elaboró un protocolo de laboratorio a partir del acompañamiento de 80 endoscopias de vías digestivas altas luego de aplicar los criterios de selección y solicitar consentimiento informado; se tomaron biopsias a 30 pacientes, las cuales se cultivaron mediante pruebas microbiológicas. La frecuencia de diagnóstico endoscópico fue de 22 casos de gastritis, un caso de ulcera péptica, tres casos de cáncer gástrico y cuatro personas asintomáticas. 20 muestras fueron compatibles con $\mathrm{H}$. pylori. Se seleccionaron cinco cepas, a las cuales se les realizó cinética de crecimiento y fueron evaluadas con diferentes pruebas bioquímicas. Se determinó que las condiciones microbiológicas utilizadas en el protocolo son idóneas para el óptimo aislamiento de H. pylori.

Palabras clave: Helicobacterias, Cepario, Cinética de crecimiento, cultivo microbiano.

1 Grupo de Investigaciones Biomédicas y de Genética Aplicada (GIBGA), Programa de Medicina, Facultad de Ciencias de la Salud, Universidad de Ciencias Aplicadas y Ambientales - U.D.C.A, Calle 222 No. 55-37, Bogotá, Colombia

2 Autor para correspondencia: andresjulian1981@gmail.com

3 Medico gastroenterólogo 


\section{ABSTRACT}

Helicobacter pylori is a microorganism that infects an estimated 50 to $70 \%$ of the general population in developing countries and is associated with infection biopsychosocial factors. Currently the pathogenic bacteria relationship with gastritis, peptic ulcer and gastric cancer, and even on this last condition is called, WHO in 1994 declared as type I carcinogen microbiological In Colombia there are few clinically characterized collections of this microorganism. This study determined the microbiological conditions for the establishment of a culture collection of H. pylori. A laboratory protocol was developed from Escort 80 upper gastrointestinal endoscopy after applying the selection criteria and request informed consent; biopsies of 30 patients which were cultured by microbiological tests were taken. The frequency of endoscopic diagnosis was 22 cases of gastritis, peptic ulcer in one case, three cases of gastric cancer and four asymptomatic persons. 20 samples were compatible with $H$. pylori. Five strains which growth kinetics was performed were selected; these were evaluated by several biochesmistry tests. It was determined that the microbiological conditions used in the protocol are suitable for optimal isolation of $\mathrm{H}$. pylori.

Keywords: Helicobacter, Protocol, growth kinetics, microbial culture.

\section{INTRODUCCIÓN}

Helicobacter pylori es una bacteria que infecta del 50 al 75\% de la población mundial. La Organización Mundial de la Salud-OMS, ha clasificado este patógeno como agente biológico carcinógeno para el hombre en la categoría 1. De acuerdo con lo reportado por Wilson \& Crabtree, 2007 en el curso de la infección, éste se adhiere a la célula epitelial, activando por un lado células dendríticas, monocitos y macrófagos y por otro induciendo una fuerte respuesta humoral en la mucosa gástrica, estableciendo un perfil inflamatorio crónico que genera daño del tejido y el posterior desarrollo de patología gástrica concomitante (Suerbaum y Michetti, 2002; O'Keeffe y Moran, 2008; Herrera y Parsonnet, 2009; Sutton y Chionh, 2013).

La infección por $H$. pylori varía entre diferentes grupos poblacionales. Se ha visto que el riesgo de infección en países desarrollados es cercano al 60\%; mientras que en países en vía de desarrollo asciende al 90\% (Navarro et al., 2007; Azevedo et al., 2007). En su biología, H. pylori se aísla a partir de muestras de mucosa gástrica o extra gástrica y para su cultivo y caracterización, requiere de medios y pruebas específicas (Whitmire y Merrell, 2012; Bayona, 2013).

H. pylori tarda aproximadamente tres a siete días en formar colonias bajo las siguientes condiciones: 5-10\% $\mathrm{O}_{2} ; 5-10 \% \mathrm{CO}_{2} ; 80-90 \% \mathrm{~N}_{2}$, humedad de $95 \%$ y temperatura de $37^{\circ} \mathrm{C}$, parámetros que se obtienen mediante el empleo de sistemas comerciales. El microorganismo se cultiva en medios suplementados con sangre de caballo, hemoglobina, suero fetal bovino, carbón vegetal o emulsión de yema de huevo; además se necesitan nutrientes como peptona, triptona, extracto de levadura, glucosa, cloruro de sodio y bisulfito de sodio para obtener un crecimiento adecuado; a estos cultivos es importante adicionar antibióticos como vancomicina, trimetropim-sulfa, 
polimixina y anfotericina B con el fin de minimizar el crecimiento de otros microorganismos (Douraghi et al., 2010; Hutton et al., 2011; Mohammadi et al., 2012; Tonkic et al., 2012; Cover, 2012; Otero, 2013; Bayona, 2013). El aislamiento y caracterización de $H$. pylori es complejo de implementar debido a las particularidades para su crecimiento y conservación, de acuerdo a lo anterior el objetivo de este trabajo fue determinar las condiciones para establecer un cepario de $\mathrm{H}$. pylori aislado a partir de biopsias tomadas de diferentes casos clínicos.

\section{MATERIALES Y MÉTODOS}

Se evaluaron muestras de biopsias de pacientes a quienes se les realizó endoscopia de vías digestivas altas y recibieron diagnóstico de gastritis, úlcera péptica, cáncer gástrico y de una persona asintomática durante el periodo de 2012 a 2013. Los pacientes asistieron a consulta privada con gastroenterólogo en dos instituciones de salud en Bogotá, Colombia.

En cuanto a la población de pacientes de quienes se obtuvo las muestras, la distribución por género fue de 16 hombres y 14 mujeres, el promedio de edad fue 54 años (20 y 83 años), cuya procedencia en la mayoría de casos fue de Bogotá con 21 pacientes, los 9 pacientes restantes, procedían de municipios aledaños a Bogotá, tales como Tocaima, Gachancipá, Ibagué, Anapoima, Sesquilé, Chipaque, El Cocuy, Iconozo y Yacopí.

Criterios de inclusión - exclusión para pacientes con sintomatología gástrica. Criterios de inclusión. Edad entre 18 y 70 años. Realización de endoscopia de vías digestiva altas (esófago gastro duodenoscopia). No haber recibido tratamiento previo para erradicación de $H$. pylori, ni medicamentos anti-secretores durante las 24 horas antes de la toma de biopsia. Diagnóstico positivo de cáncer gástrico, úlcera o gastritis establecido por Gastroenterólogo. Haber firmado consentimiento informado y autorización para la toma de muestra.
Criterios exclusión. Presentar enfermedades sistémicas severas, patologías adicionales a las evaluadas a nivel de esófago, estómago o duodeno, o presentar patologías como Inmunosupresión. Recibir terapia de anticoagulación o presentar hemorragia gastrointestinal activa o sangre en la cavidad gástrica. No aceptar participar en el estudio.

Criterios de inclusión - exclusión en individuos asintomáticos

Criterios de inclusión. Pacientes en edades entre 18 y 70 años. Presentar enfermedades sistémicas severas o patología a nivel de esófago, estómago o duodeno. No haber recibido tratamiento previo para erradicación de H. pylori, ni medicamentos anti-secretores durante las 24 horas antes de la toma de biopsia. Haber firmado consentimiento informado y autorizaciones de toma de muestra.

Criterios de exclusión. Presentar enfermedades concomitantes como insuficiencia renal crónica, insuficiencia cardiaca descompensada, insuficiencia respiratoria, tumores, enfermedades malignas. Haber recibido cirugía gástrica previa. Mujeres en embarazo o lactantes. Tener diagnóstico de enfermedades psiquiátricas. Presentar adicciones a drogas o alcohol.

Procedimientos clínicos y microbiológicos para aislamiento de cepas. Criterios para la obtención de muestras. Los criterios endoscópicos fueron mucosa enrojecida con imágenes endoscópicas eritematosas compatible con gastritis, lesión tipo ulcera péptica compatible con ulcera, lesión tipo tumoral, coliflor o lisa, herniación de la mucosa sangrante o no sangrante compatible con cáncer gástrico. Cada caso clínico presentó un registro del diagnóstico endoscópico por parte del especialista, se adjuntó la lista de criterios, una breve historia clínica con indicación endoscópica y por último, el consentimiento informado (Yantiss y Odze, 2009; Hutton et al., 2011; Mohammadi et al., 2012; Tonkic et al., 2012; Cover, 2012). 
Se realizó endoscopia de vías digestivas altas en consulta privada del Gastroenterólogo. Se empleó un equipo Olympus Evis Exera CLE-145 Score, CV-145 y Endoscopio GIF Olympus Tips $\vee$ y biopsias tomadas con pinzas Olympus FB-21K. Los endoscopios y las pinzas fueron desinfectados el día anterior a la toma de muestra con glutaraldehído al $3 \%$ y fueron lavados posteriormente con agua destilada estéril. Las endoscopias para la toma de biopsias se realizaron de acuerdo con el protocoló de Sídney del 2009 (Yantiss y Odze, 2009). Todos los procedimientos clínicos se realizaron bajo consentimiento informado y el presente proyecto fue aprobado por el comité de ética de la Universidad de Ciencias Aplicadas y Ambientales.

Procedimientos microbiológicos. Dos de las biopsias frescas extraídas del paciente se depositaron en medio liquido Campylobacter adicionado con sangre de caballo al $7 \%$, isovitalex al $2 \%$ y antibióticos selectivos: trimetropim-sulfametozazol $(0,1 \%)$, anfotericina $b(0,1 \%)$, penicilina $(0,1 \%)$, vancomicina (0 01\%) y fueron transportadas en una nevera estéril portátil a $8^{\circ} \mathrm{C}$ a las instalaciones de la U.D.C.A dentro de las primeras seis horas (Yantiss y Odze, 2009; Mohammadi et al., 2012).

Caldos primarios. A partir de las muestras trasportadas, se extrajeron $200 \mu \mathrm{L}$ de sobrenadante y se realizó la prueba rápida de ureasa, luego se taparon los tubos y se empaquetaron de forma vertical en la bolsa de plástico (Gaspak ${ }^{\circledR}$ pouch BBL) más el dispositivo de anaerobiosis (Gaspak ${ }^{\circledR}$ ) cerrándose herméticamente. Se llevaron a la incubadora durante 5 días a $37^{\circ} \mathrm{C}$ bajo las siguientes condiciones: $\mathrm{CO}_{2} 5-10 \%$; $\mathrm{O}_{2}$ 5-10\%; $\mathrm{N}_{2}$ 80-90\%, humedad de 95\% (Cover, 2012; Otero, 2013; Bayona, 2013).

Identificación de aislamientos de $H$. pylori. Después de cinco días, se tomó una muestra directamente de los caldos primarios con asa bacteriológica estéril y se realizaron subcultivos en agar Brucella y Columbia suplementado con Isovitalex, sangre de caballo al 7\% y antibióticos selectivos.
Posteriormente, las muestras se llevaron nuevamente a condiciones de microaerofilia de acuerdo a lo mencionado por Bayona, 2013. Finalmente, a los aislamientos se le realizaron las pruebas bioquímicas: ureasa (Corpogen), catalasa y citocromo-oxidasa. Los aislamientos compatibles con $\mathrm{H}$. pylori fueron aquellos positivos para estas tres bioquímicas. Estas pruebas se realizaron tanto desde colonia como desde una suspensión precipitada del caldo Campylobacter durante tres subcultivos sucesivos (Navarro et al., 2007; Whitmire y Merrell, 2012; Bayona, 2013).

Cinéticas de crecimiento de los aislamientos compatibles con $\mathrm{H}$. pylori. Para la determinación de la cinética de crecimiento se registró la relación del volumen bacteriano cuantificado por densidad óptica mediante el uso del espectrofotómetro frente al tiempo de seguimiento. Como patrón de comparación se tomó la cepa de referencia NCTC 11638 donada por el laboratorio de microbiología de la Pontificia Universidad Javeriana al grupo de investigación GIBGA.

Los aislamientos fueron crecidos en agar Brucella adicionados con sangre equina desfibrinada estéril al $7 \%$, isovitalex al $2 \%$ y extracto de levadura al $1 \%$. Una vez se observaron colonias estas fueron suspendidas en medio líquido Campylobacter y se compararon con el tubo número tres de la escala de MacFarland equivalente a $1 \times 10^{8}$ microorganismos y fueron incubadas a $37^{\circ} \mathrm{C}$ en condiciones de microaerofilia empleando sobres Gaspack - 5\% $\mathrm{O}_{2}, 5-10 \%$ $\mathrm{CO}_{2}, 80-90 \% \mathrm{~N}_{2}$, humedad 95\%. Las muestras fueron evaluadas por triplicado.

Se realizaron mediciones de la densidad óptica a $550 \mathrm{~nm}$ cada 24 horas por espacio de tres días y como control negativo de lectura, se empleó medio líquido Campylobacter sin inocular. Se tomaron a partir de cada cepa, cuatro tubos Falcón conteniendo medio liquido Campylobacter + isovitalex (1\%) (Volumen final $6 \mathrm{~mL}$ ) los cuales fueron incubados en microaerofilia a $37^{\circ} \mathrm{C}$ para ser evaluados (Alarcón et al., 2004; Blanchard y Nedrud, 2012). 
Análisis estadístico: Para las variables cepa, pruebas microbiológicas y frecuencia diagnóstica se empleó un análisis de frecuencias absolutas para cada una de los posibles valores. Para las variables cuantitativas como número de biopsias y edad, se realizaron estadísticas descriptivas determinando las medidas de tendencia central. Para el análisis de la cinética de crecimiento de las cepas, donde se relacionó crecimiento y tiempo, inicialmente se realizó la prueba de Friedman considerando significativo diferencias con un valor $\mathrm{p}<0,05$; posteriormente se utilizó la prueba de Wilcoxon para determinar diferencia en el crecimiento en rangos de tiempo considerando un valor $p<0,025$ de significancia asintótica bilateral como estadísticamente significativo y finalmente la prueba $U$ de Mann-Whitney, la cual analizó el crecimiento de dos cepas especificas en un rango de tiempo, considerando diferencias estadísticamente significativas valores con $p<$ 0,05 . Los análisis se realizaron mediante paquete estadístico IBM SPSS Statistics versión 22.01.

\section{RESULTADOS Y DISCUSIÓN}

En el presente estudio se obtuvieron 20 aislamientos positivos para $H$. pylori por caracterización microbiológica de un total de 30 muestras procesadas, lo que corresponde a un aislamiento efectivo en el $67 \%$ de los casos.

A nivel latinoamericano se encontraron resultados concordantes, como son el caso de Arismendi et al. (2013) quienes lograron un aislamiento en el $70 \%$ de los casos. Chilihua et al. (2003) consiguieron aislar la bacteria en el 66,7\% de las 27 muestras que incluyó en su estudió. En Colombia en el año 2010, la Universidad Javeriana reportó un aislamiento del $80 \%$ en un estudio que incluyo a 99 pacientes utilizando como medio de aislamiento de H. pylori, agar Wilkins-Chalgren (Trespalacios et al., 2010), adicionalmente en el Instituto Nacional de Cancerología, en el año 2005, obtuvieron aislamientos a partir de 166 casos empleando el medio Campylobacter (Quiroga et al., 2005). En un estudio Ilevado a cabo por He et al. (2002), que evaluó 85 biopsias al azar, se detectó H. pylori en el $89 \%$ de los casos, mediante Q-PCR.

Al describir la frecuencia de las patologías evaluadas, el diagnóstico más frecuente correspondió a la gastritis (73\%) seguida de mucosa sana (14\%) y en tercer lugar, cáncer gástrico (10\%). Mediante la caracterización microbiológica se determinó la presencia de $H$. pylori con relación a las diferentes patologías ya mencionadas. La efectividad del aislamiento de la bacteria fue de $64 \%$ a partir de muestras de gastritis y un $100 \%$ a partir de mucosa sana; así mismo 64\% y $67 \%$ obtenidas de muestras de cáncer gástrico y úlcera péptica, respectivamente.

Por otro lado, un hallazgo interesante del presente estudio fue que la cinética demostró diferencias significativas en el crecimiento de los aislamientos. El crecimiento de las cepas provenientes de gastritis eritematosa antral, gastritis erosiva aguda y crónica antral, adenocarcinoma, linfoma gástrico y la cepa de referencia fueron diferentes (valor $p$ de 0.001). Sin embargo, este crecimiento no está vinculado a una patología especifica, es decir, aislamientos obtenidos de dos pacientes con gastritis presentaron crecimientos diferenciales. Esto podría sugerir que las cepas de un paciente con infección múltiple podrían tener rangos de crecimiento diferentes (Figura 1).

En la actualidad no existen muchos reportes en los que se compare el crecimiento de cepas provenientes de diferentes patologías o que hayan correlacionado esta variable; por otra parte existen diferentes reportes que comparan medios líquidos entre si y otros que han descrito el crecimiento de cepas puntuales en medios líquidos (Douraghi et al., 2010).

En la cuantificación a las 24 horas se observó un crecimiento de todas las cepas, en orden descendente el cambio en las densidades ópticas (OD) fue: 


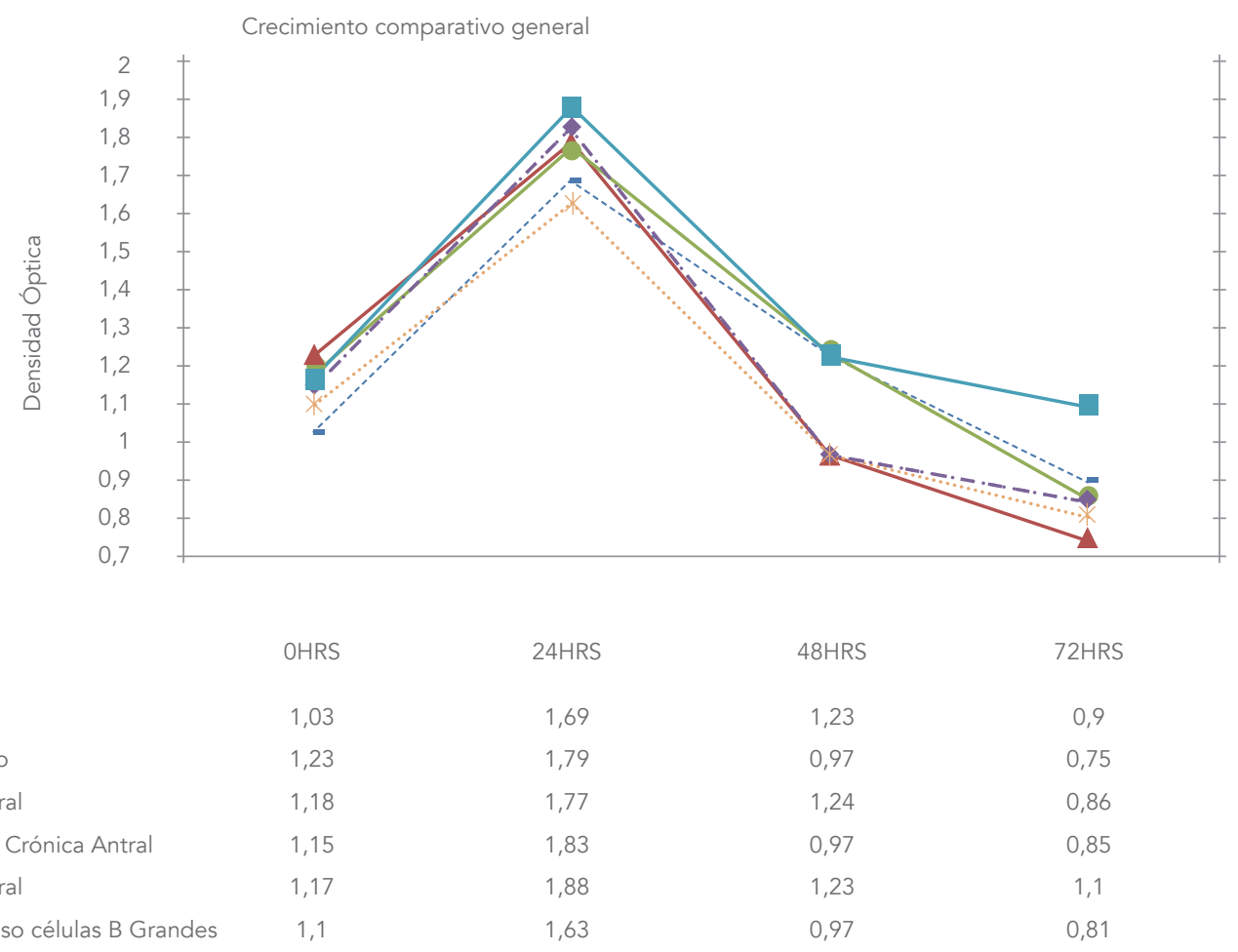

Figura 1. Cinéticas de crecimiento de las muestras seleccionadas. Cada línea representa la cinética de crecimiento de una cepa aislada de diferentes patologías. Los tiempos de evaluación fueron 0, 24, 48 y 72 horas (HRS). La población bacteriana se medió en densidades ópticas (DO).

gastritis eritematosa antral $(0,710 \mathrm{D})$, gastritis erosiva aguda y crónica antral (0,68 OD), NCTC 11638 $(0,660 D)$, gastritis eritematosa antral (0,59 OD), adenocarcinoma gástrico (0,56OD) y finalmente linfoma no hodgkin difuso células $B$ grandes con 0,53 OD.

Las cinéticas de crecimiento empezaron a decrecer a las 48 horas mostrando el siguiente patrón en orden descendente: gastritis erosiva aguda y crónica antral (-0,86 OD), adenocarcinoma gástrico (-0,82 OD) Linfoma no hodgkin difuso células B grandes con (-0,66 OD). Gastritis Eritematosa antral $(-0,60 D)$, gastritis eritematosa antral $(-0,530 D)$ y finalmente cepa control (-0,460D).

El comportamiento de la cinética de crecimiento comparando cepas provenientes de diferentes patologías fue similar, excepto la cepa proveniente de la gastritis eritematosa antral que presentó un mayor crecimiento a lo largo de las 72 horas. Las cepas provenientes de adenocarcinoma gástrico y gastritis eritematosa aguda y crónica antral fueron las segundas cepas de mayor crecimiento (mostrando un comportamiento similar), seguida de la cepa proveniente de la gastritis eritematosa antral. La cepa de referencia fue la segunda cepa de menor crecimiento, y por último, la cepa proveniente de linfoma gástrico fue la de menor crecimiento a lo largo de las 72 horas.

Seguidamente, se observó un crecimiento inmediato superior por parte del medio Campylobacter sobre 5\% FBS (suero fetal bovino), 0,2\% b-cyclodextrina y BB (Brucella) teniendo en cuenta que a las 24 horas la diferencia de OD oscilaron entre 0,56 y 0,71 en medio Campylobacter; en cambio en los otros 
tres cultivos la diferencia de OD a las 24 horas osciló entre 0,1 y 0,6; siendo mayor en el medio liquido Campylobacter, pero en otro aspecto el decrecimiento fue mayor en Campylobacter ya que a las 48 horas entró en disminución de las OD; los valores oscilaron entre $-0,46$ y $-0,82$ a diferencia de los otros tres medios, los cuales a las 48 horas en cuatro cepas aún existió periodo de crecimiento que osciló entre 0,2 a 0,6 OD, las otras dos cepas mantuvieron un periodo de meseta (Douraghi et al., 2010). Finalmente a las 72 horas las cepas entraron en un periodo de decrecimiento mayor, las OD oscilaron entre $-0,12$ y $-0,38$ (Douraghi et al., 2010).

Finalmente, comparando los resultados aquí mostrados con el del trabajo de Joo et al. (2010) donde se estudió el crecimiento de las cepas en caldo Brucella suplementado con suero de caballo al 10\% a intervalos de cuatro horas hasta completar 40 horas reveló la OD a las 24 horas era de 2,7 y de 1,9 en dos diferentes condiciones de concentraciones de $\mathrm{CO}_{2}$ y $\mathrm{O}_{2}$, comparado con la actual cinética muestra un crecimiento inferior por parte del medio Campylobacter.

\section{AGRADECIMIENTOS}

Los autores expresan su agradecimiento a la Universidad de Ciencias Aplicadas y Ambientales U.D.C.A, por su apoyo en la financiación del presente trabajo. Conflicto de intereses: El artículo fue preparado y revisado con la participación de todos los autores, quienes declaramos que no existe conflicto de intereses que ponga en riesgo la validez de los resultados presentados.

\section{REFERENCIAS}

1. ALARCÓN, T.; BAQUERO, M.; DOMINGO, D.; LÓPEZ, M.; ROYO, G. 2004. Diagnóstico microbiológico de la infección por Helicobacter pylori. Recomendaciones de la Sociedad Española de Enfermedades Infecciosas y Microbiología Clínica. España. 25p.

2. AMIEVA M.; OMAR E. 2008. Host Bacterial Interactions in Helicobacter pylori Infection. Gastroenterology. 134(1):306-329.

3. ARISMENDI, G.; HERNÁNDEZ, I.; MENGUAL, E.; ABREU, N.; MOLERO, N.; FUENMAYOR, A.; ROMERO, G.; LIZARZÁBAL, M. 2013. Estimación de riesgo de cáncer gástrico en pacientes con gastritis crónica asociada a la infección por Helicobacter pylori en un escenario clínico. Rev. Gastroenterol. Mex. 78(3):135-43.

4. AZEVEDO, N.; GUIMARAES, N.; FIGUEIREDO, C.; KEEVIL, C.; VIEIRA, M. 2007. A New model for the transmission of Helicobacter pylori: Role of environmental reservoirs as gene pools to increase strain diversity. Critical Rev. Microbiol. (Inglaterra). 33(3):157-169.

5. BAYONA, M. 2013. Condiciones microbiológicas para el cultivo de Helicobacter pylori. Rev. Col. Gastroenterol. Bogotá.28(2):94-99.

6. BLANCHARD, T.; NEDRUD, J. 2012. Laboratory maintenance of Helicobacter species. Current Protocols Microbiol. Supplement 24: 23p.

7. CHILIHUA, K.; PALOMINO, R.; AGUILAR., E. 2003. Aislamiento de Helicobacter pylori a partir de biopsias gástricas de pacientes con gastritis en el Hospital Regional del Cusco, Perú. SITUA. 13(1):15-19. 
8. COVER, T. 2012. Perspectives on methodology for in vitro culture of Helicobacter pylori. Methods Mol. Biol. 921:11-15.

9. DOURAGHI, M.; KASHANI, S.; ZERAATI, H.; ESMAILI, M.; OGHALAIE, A.; MOHAMMADI, M. 2010. Comparative evaluation of three supplements for Helicobacter pylori growth in liquid culture. Current Microbiol. 60(4):254-262.

10. HE, Q.; WANG, J.; OSATO, M.; LACHMAN, L. 2002. Real-time quantitative PCR for detection of Helicobacter pylori. J. Clin. Microbiol. 40(10):3720-3728.

11. HERRERA, V.; PARSONNET, J. 2009. Helicobacter pylori and gastric adeno-carcinoma. Clin. Microbiol. Infect. 15:971-976.

12. HUTTON, M.; KAPARAKIS-LIASKOS, M.; FERRERO, R. 2011. The use of AlbuMAXII as a blood or serum alternative for the culture of Helicobacter pylori. Helicobacter. 17:68-76.

13. JOO, J.; PARK, K.; SONG, J.; KIM, D.; LEE, K.; RHEE, K. 2010. A thin-layer liquid culture technique for the growth of Helicobacter pylori. Helicobacter. 15(4):295-302.

14. MOHAMMADI, M.; HASHANI, S.; GAROOSI, Y.; TALEBKHAN, T.; JAHANGITI, S. 2012. In vivo measurement of Helicobacter pylori. Meth Mol Biol. 921: 239-49.

15. NAVARRO, J.; PEREA, A.; PINEDA, J.; DÍEZ, O.; MERCADO, M.; TRESPALACIOS, A. 2007. Evaluación de la productividad de tres medios de cultivo para la recuperación de Helicobacter pylori. Universitas Scientiarum, Rev. Fac. Cienc. (Colombia).12:79-86.

16. O'KEEFFE, J.; MORAN, A. 2008. Conventional, regulatory, and unconventional $\mathrm{T}$ Cell in the immunology response to Helicobacter pylori. Helicobacter. 13:1-19.

17. OTERO, W. 2013. La importancia de cultivar Helicobacter pylori. Rev. Col Gastroenterol. 28(2):87-92.

18. QUiROGA, A.; CITTELLY, J.; BRAVO, M. 2005. Frecuencia de los genotipos babA2, oipA y cagE de Helicobacter pylori en pacientes colombianos con enfermedades gastroduodenales. Biomédica. (Colombia). 25(3):325-334.

19. SUERBAUM, S.; MICHETTI, P. 2002. Helicobacter pylori infection. N. Engl. J. Med. (Estados Unidos). 347:1175-1186.

20. TONKIC, A.; TONKIC, M.; LEHOURS, P.; MEFRAUD, F. 2012. Epidemiology and diagnosis of Helicobacter pylori infection. Helicobacter.; 17(Suppl. 1):1-8.

21. TRESPALACIOS, A.; OTERO, W.; MERCADO, M. 2010. Resistencia de Helicobacter pylori a metronidazol, claritromicina y amoxicilina en pacientes colombianos. Rev. Col. Gastroenterol. 25(1):31-38.

22. WHITMIRE, J.; MERRELL, D. 2012. Successful culture techniques for Helicobacter Species: general culture techniques for Helicobacter pylori. Meth. Mol. Biol. 921:17-27.

23. WILSON, K.; CRABTREE, J. 2007. Immunology of Helicobacter pylori: insights into the failure of the immune response and perspectives on vaccine studies. Gatroenterology. 133:288-308.

24. YANTISS, R.; ODZE R. 2009. Optimal approach to obtaining mucosal biopsies for assessment of inflammatory disorders of the gastrointestinal tract. AJG. (Estados Unidos). 104:774-783. 